\title{
Fault Detection of a Wind Turbine's Gearbox, based on Power Curve Modeling and an on-line Statistical Change Detection Algorithm
}

\author{
Basheer W. Shaheen ${ }^{1}$, Ahmed Abu Hanieh ${ }^{2}$, István Németh ${ }^{1}$ \\ ${ }^{1}$ Department of Manufacturing Science and Engineering, Budapest University of \\ Technology and Economics, Múegyetem rkp. 3, 1111 Budapest, Hungary, \\ shaheen.basheer@gpk.bme.hu, nemeth.istvan@gpk.bme.hu \\ ${ }^{2}$ Department of Mechanical and Mechatronics Engineering, Birzeit University, \\ 97200, Palestine, ahanieh@birzeit.edu
}

\begin{abstract}
An early model-based fault detection was developed, based on the wind turbine's power curve to detect the degradation (faults) in gearbox efficiency, resulted from the existing mechanical losses (torque losses) through the low-speed shaft and the high-speed shaft, then to assist in implementing predictive maintenance strategy. The detection was performed on two levels; the first level represents a slight and progressive degradation in the gearbox efficiency. The other one represents a radical (abrupt) degradation in the efficiency. Artificial SCADA data for different measurements (wind speed and active power) in both fault-free and faulty operating modes were generated using a FAST-NREL simulator. The wind turbine power curves' parameters were estimated, then power residuals were generated from each power point. Finally, an on-line CUSUM statistical change detection algorithm was used to evaluate and detect small changes in power residuals generated from the model. The presented fault detection system successfully detected faults in both detection levels under realistic wind turbulence and with a fault magnitude of $2 \%$ efficiency degradation for the progressive degradation level.
\end{abstract}

Keywords: fault detection; SCADA; wind turbine; condition monitoring; change-point detection; simulation; optimization

\section{Introduction}

Currently, wind energy is one of the primary renewable energy sources used to generate electrical power. Experts expect that more than 355 Gigawatts of new capacity will be added between 2020 and 2024 [1]. This energy source plays a key role in reducing the harmful effects of other, traditional electrical power generation methods, such as, fossil fuels, coal and natural gas, which contribute to global warming and have increased the levels atmospheric carbon dioxide (CO2) [2]. 
Electricity generation using wind and wind turbines, is considered to be one of the cleanest, environmentally friendly, electricity generation methods, accepted by society and has numerous competitive economic advantages [3] [2]. The efficiency of the power generation from a Wind Turbine (WT) could be increased by better controlling the wind turbine's operations, according to the information related to wind state changes and the turbine location [4].

The wind energy conversion system is known as a Wind Turbine. A wind turbine can be defined as "a rotating mechanical device that converts wind kinetic energy to practical mechanical energy, resulting in electricity production" [5]. Wind turbines have two common types; horizontal axis wind turbines (HAWT) and vertical axis wind turbines (VAWT) [6] [7]. According to [6], the most used design is HAWT, for which the axis of rotation is parallel to the grounds surface, as shown in Figure 1.

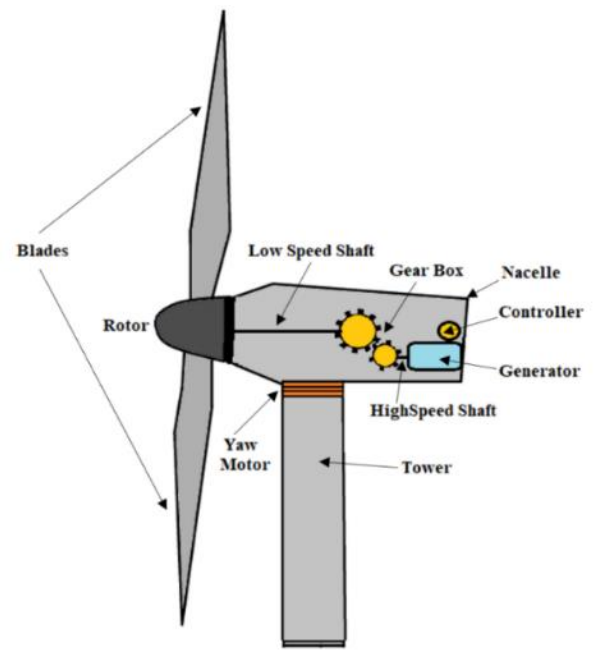

Figure 1

HAWT main components

The main goal of this research is to develop an early, model-based, fault detection method and to monitor the performance degradation of WTs (faults through the gearbox) based on the WT's power curve, using artificial (simulated) supervisory control and data acquisition (SCADA) data (typically sampled at low frequency). This performance degradation (power loss) is typically due to the degradation in the Gear Box Efficiency (GBoxEff) resulted from the existing mechanical losses (torque losses) through the low-speed shaft (LSS) and the high-speed shaft (HSS). This model can be enhanced by a successful implementation of a predictive maintenance strategy, to reduce the associated maintenance costs and produce better maintenance planning and scheduling activities. 
A polynomial model and the least-squares algorithm for polynomial parameters estimation were used as a model structure. Model-based fault detection was performed for the power residual generated from the model and using residuals' evaluation modules, based on CUSUM statistical change detection algorithm and for two different levels of gearbox efficiency degradation. The first degradation level was for a progressive (slight) degradation in GBoxEff, and the other level represented a radical or abrupt degradation in the efficiency.

\section{Literature Review}

According to Tchakoua et al. [8], the operations and maintenance (O\&M) costs of wind turbines farm are supposed to be higher than the traditional power generation methods due to the remote locations of such farms, the bad weather conditions, and other variables. Thus, the development of fault diagnosis systems to provide an early warning of faults and prediction, with health condition monitoring systems, are needed to reduce the O\&M costs [9].

Most of the research papers focused on condition monitoring systems (CMS) tools to diagnose faults and monitor the health of wind turbines, which include "sensors, signal acquisition, and processing software, cabling and installations that gives continuous information about the monitored component condition" [5]. CMS is used in offshore wind turbines, especially, to monitor the most critical components in the WT system, such as gearbox, generator, rotor blades, and yaw actuator [5] [8]. To determine the portion of each the WT components, out of the total number of failures that occurred, Hahn et al. [10] reported a survey of 1500 WTs over a period of 15 years that indicated five component groups, "electrical system, control system, hydraulic system, sensors, and rotor blades" are responsible for $67 \%$ of the failures that occurred.

Different data analysis and data mining algorithms such as Fuzzy logic, artificial neural network (ANN), support vector machine (SVM), neural network, and deep belief networks were used in many research works to detect/predict the faults and to monitor the health conditions of the WTs [11-15].

In order to develop a WT's CMS, we need data to validate the model; in modern WTs, SCADA data systems are commonly used [16]. SCADA systems for data analysis of WTs condition monitoring (CM) are cost-effective, reliable, and practical [17]. The principle of a SCADA system is based on collecting extensive information from key WT subassemblies using sensors installed on the WT [8]. The operational data of a WT usually indicate either the WT status or measurement signals, such as wind speed, temperature, power, and current, which reflect the real-time condition of the WT components. By analyzing the SCADA data, these signals and the different relationships between them can be observed, and the condition (health) of the WT can be concluded [18]. Tao et al. 
[19] used an efficient SCADA data mining method to detect anomalies of the wind turbines using combinations of data pre-processing algorithms such as grey correlation algorithm to extract the eigenvectors of the monitored data. Support vector regression (SVR) parameters were optimized through the genetic algorithm and cross-validation method, resulting in a high precision fault prediction model.

Similarly, Corley et al. [20] predicted the anomalies from measured SCADA data in wind turbine gearboxes using combined thermal modeling and machine learning method.

In Schlechtingen et al. [21] [22], a wind turbine condition monitoring (WTCM) system based on SCADA data, using normal behavior models and adaptive neuro-fuzzy inference systems (ANFIS) was presented. It is worth mentioning that it is difficult to detect a fault from raw SCADA data without using an appropriate data analysis tool [22]. Another research work by Yang et al. [23] focused on developing a cost-effective and reliable CM (technique) for WT blades and drivers through the interpretation of SCADA data collected from a farm of WTs. In Zaher et al. [24] and Zhang and Wang [25], different techniques for anomaly detection in WTs based on SCADA data using an ANN were used. The results indicated that the proposed techniques for SCADA data interpretation could identify the early faults and give a WT performance assessment to give the system's operator sufficient time to make the needed decisions concerning the machine maintenance process.

Similarly, Godwin and Matthews [26] proposed a data-driven system to classify the faults associated with WT pitch through SCADA data. Data were collected from 8 WTs every 10 minutes over a period of 28 months. Another research work of Kusiak and $\mathrm{Li}$ [27] used many data mining algorithms to develop a fault detection and identification (FDI) system including many WT's components; fault data were obtained by SCADA data system, and fault prediction was applied through 3 levels, which include fault-no fault prediction, fault classification into categories and prediction of specific types of faults; fault prediction model in this study was developed using different types of data mining algorithms including, the Neural Network (NN), the Standard Classification and Regression Tree (CART), the Boosting Tree Algorithm (BTA), and the Support Vector Machine (SVM). Likewise, Yuan et al. [9] presented a wind turbine gearbox fault prediction approach based on SCADA data. The normal conditions of the WT were modeled using a stacking model consisted of Random Forest (RF), Gradient Boosting Decision Tree (GBDT), and Extreme Gradient Boosting (XGBOOST). The Mahalanobis distance (MD) method instead of the residual generation was used to measure the deviation of the current conditions from the normal conditions of the turbines. Lastly, a change-point detection algorithm based on cumulative summation (CUSUM) was used to predict the resulted faults. 
Likewise, Borchersen and Kinnaert [28] proposed and tested an early modelbased fault detection for the cooling system of WT's generator. In this study, the model parameters were estimated on-line using an extended Kalman filter, and then residuals were evaluated by CUSUM statistical change detection algorithm in order to detect the small faults. Another related research by Pujol-Vazquez et al. [29] developed an innovative approach to detect faults in the WT's pitch actuator system based on the interval observer framework theory, which has been proved to be an efficient method to measure the uncertainties in dynamic systems.

In general, fault detection based on SCADA data can be conducted using different modeling and analysis techniques as presented in the previous works.

\section{Proposed Fault Detection Framework}

Figure 2 below presents the overall framework used to detect/predict the gearbox faults in different efficiency degradation levels. It is composed of three main phases. The first phase is the Artificial SCADA data simulation/generation, in which SCADA data for different meteorological and mechanical conditions are simulated, then raw SCADA points are generated. The second phase is the modeling phase. It contains (i) the data pre-processing where outlying and unnecessary data are filtered from the raw SCADA data; (ii) the power curve construction that is based on the generated power and wind speed; and (iii) the parameters estimation that uses an appropriate regression method. Through the application phase, power residuals are generated based on the healthy and faulty processed SCADA data and for different levels of gearbox efficiency degradation. Then, the estimated model is partially validated based on the least mean squared error. Finally, the change-point detection based on CUSUM algorithm (recursive form) is applied to implement the fault detection/prediction model. 


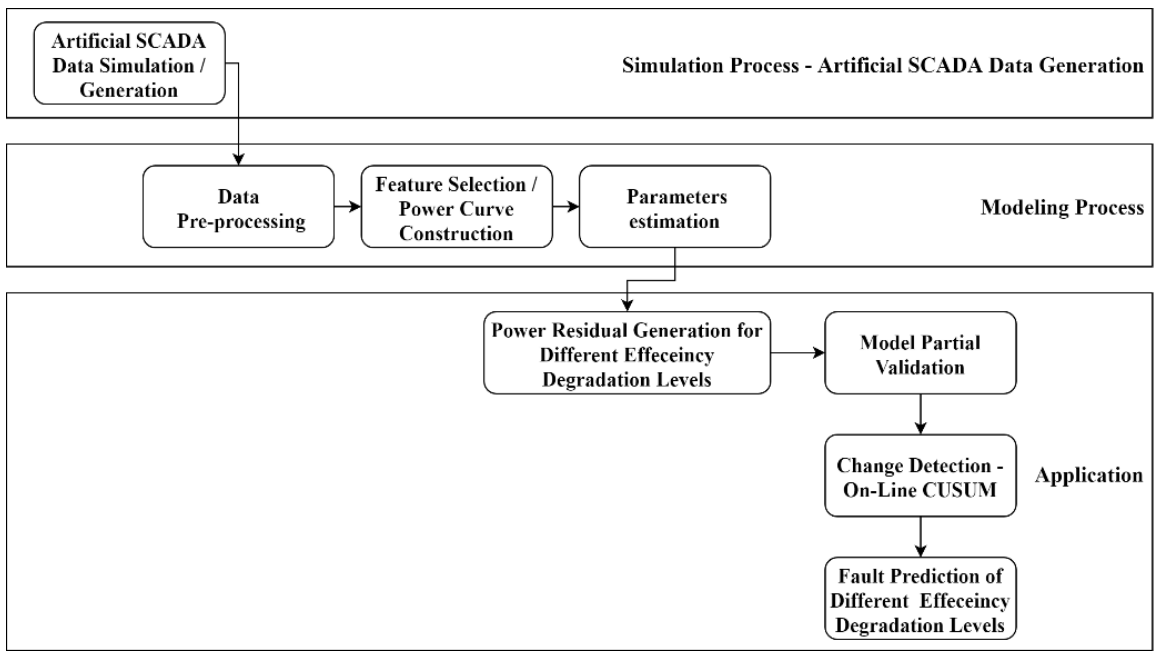

Figure 2

Fault detection framework

\subsection{Artificial SCADA Data Simulation}

\subsubsection{The Wind Turbine Simulator}

The wind turbine simulator used in this research consisted of a set of codes that were developed by NREL (USA Government based, National Renewable Energy Laboratory). For example, the AeroDyn code conducts aerodynamic calculations while the FAST code conducting the aeroelastic simulation. Both together simulate the wind turbine's mechanical behavior. The control scheme of all operations of the wind turbine has been added to the aforementioned two codes through SIMULINK in MATLAB. The needed wind input files to AeroDyn and FAST codes were modeled and generated through TurbSim software depending on the required input parameters such as mean wind speed ad turbulence intensity (TI) [30-32].

\subsubsection{Simulation Basic Characteristics}

For the simulation process, meteorological conditions were set as illustrated in Table 1. Table 2 shows the main control parameters used during the simulations.

Table 1

Meteorological conditions for the simulations

\begin{tabular}{|c|c|}
\hline Turbulence Model & IECVKM=Kaimal \\
\hline IEC standard & $1-E D 2$ \\
\hline Turbulence intensity percent & $10-20 \%$ \\
\hline Wind profile type & Normal \\
\hline
\end{tabular}




\begin{tabular}{|c|c|}
\hline Height of reference wind speed & $84.672 \mathrm{~m}$ \\
\hline Mean of the wind speed at the reference height & $2-18 \mathrm{~m} / \mathrm{s}$ \\
\hline Power law exponent & 0.3 \\
\hline
\end{tabular}

Table 2

Main simulation control parameters

\begin{tabular}{|c|c|}
\hline Total Run time per point & 12 minutes \\
\hline Module step time & 0.005 seconds \\
\hline Sampling Frequency & $200 \mathrm{~Hz}$ \\
\hline
\end{tabular}

\subsubsection{Artificial SCADA Data Generation}

Two training data sets consisting of simulated (artificial) SCADA are required. Fault-free and faulty operating modes are the labels of each training set for different gearbox efficiencies. WT's fault-free (healthy) operating mode means that the WT is assumed to be operated with no mechanical losses (torque losses), being transmitted through the gearbox; thus, the GBoxEff (gearbox efficiency) is set to $100 \%$. While to simulate losses (the faulty operating mode), the WT is assumed to be operated with mechanical losses (torque losses) being transmitted through the gearbox if the GBoxEff is less than 100\%. Thus, the GBoxEff is set to $99,98,97$, and $90 \%$ to detect slight and progressive degradation in the efficiency (100-99-98-97\% respectively) and radical degradation in the efficiency (100-90\% at once). When generating power, the FAST method determines the HSS torque according to equation 1 [30].

HSS torque $=\frac{\text { LSS torque } \cdot \text { efficiency }}{\text { gear box ratio }}$

When motoring, the FAST method computes the torque on the LSS according to equation 2.

$$
\text { LSS torque }=\frac{\text { HSS torque } \cdot \text { gear box ratio }}{\text { efficiency }}
$$

It is worth mentioning that the simulated wind turbine in this research is from the type of "DeWindD6" with $1250 \mathrm{~kW}$ rated electrical power.

\subsubsection{DeWindD6 Power Curve Characteristics}

The power curve of DeWindD6 WT is illustrated in Figure 3, which depicts the power curve of DeWindD6 WT and features three key wind speeds.

Where the cut-in wind speed is the wind speed, at which the WT starts generating power, nominal wind speed is the wind speed at which the WT reaches the nominal power output, knowing that it is possible to generate higher power output above the nominal wind speed, but the control system maintains a constant power to limit loads and stresses on WT's blades. 


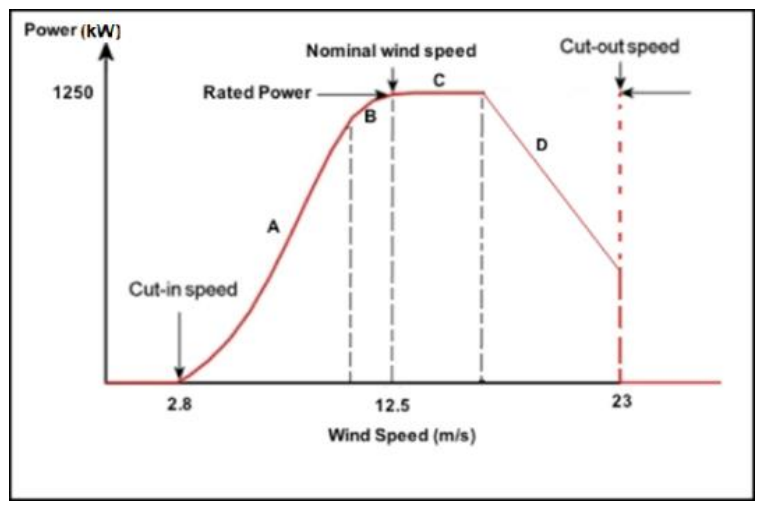

Figure 3

Nominal power curve of DeWindD6 WT

Lastly, the cut-out wind speed is the highest wind speed at which the WT can operate [33].

\subsection{Data Pre-Processing}

The resulted mean wind speed $\left(w_{j}\right)$ is a 10 minute average wind speed out of 12 minutes of total run time, where the first two minutes were assumed to be the transient time of the simulated measurement data (i.e., power and wind speed); thus, corresponding data were removed.

\subsection{Power Curve Construction}

For each simulated measurement $(j)$, the scalar average wind speed $\left(w_{j}\right)$ was calculated according to equation 3 .

$$
w_{j}=\frac{1}{n} \sum_{i=1}^{n} w_{i}
$$

Where $\left(w_{i}\right)$ is the wind speed generated by the simulator with time step 0.005 seconds. The same procedures were used to calculate the corresponding output power $(p)$. The power curve consists of primarily two input variables: wind speed and power output. Using FAST-AeroDyn simulator from NREL, and following the simulation characteristics of DeWindD6 WT, raw artificial SCADA data (active power), typically sampled at low frequency: from $30 \mathrm{~s}$ to 10 minutes, were generated along with wind speed required to construct the power curve.

According to IEC 61400-1 standard, the wind speed of the power curve is "the undisturbed free-stream wind speed at hub height, normalized for a certain air density" [34]. Once the simulation process was complete, 10 minutes' averages of wind speed and active power were generated for 1000 points for every single GBoxEff percentage. 
Figure 4 depicts a sample scatter plot of wind speed characterized by mean wind speed against active power output (i.e., the power curve) for both fault-free $(\mathrm{GBoxEff}=100 \%)$ and faulty operating modes (i.e., GBoxEff $=90 \%)$.

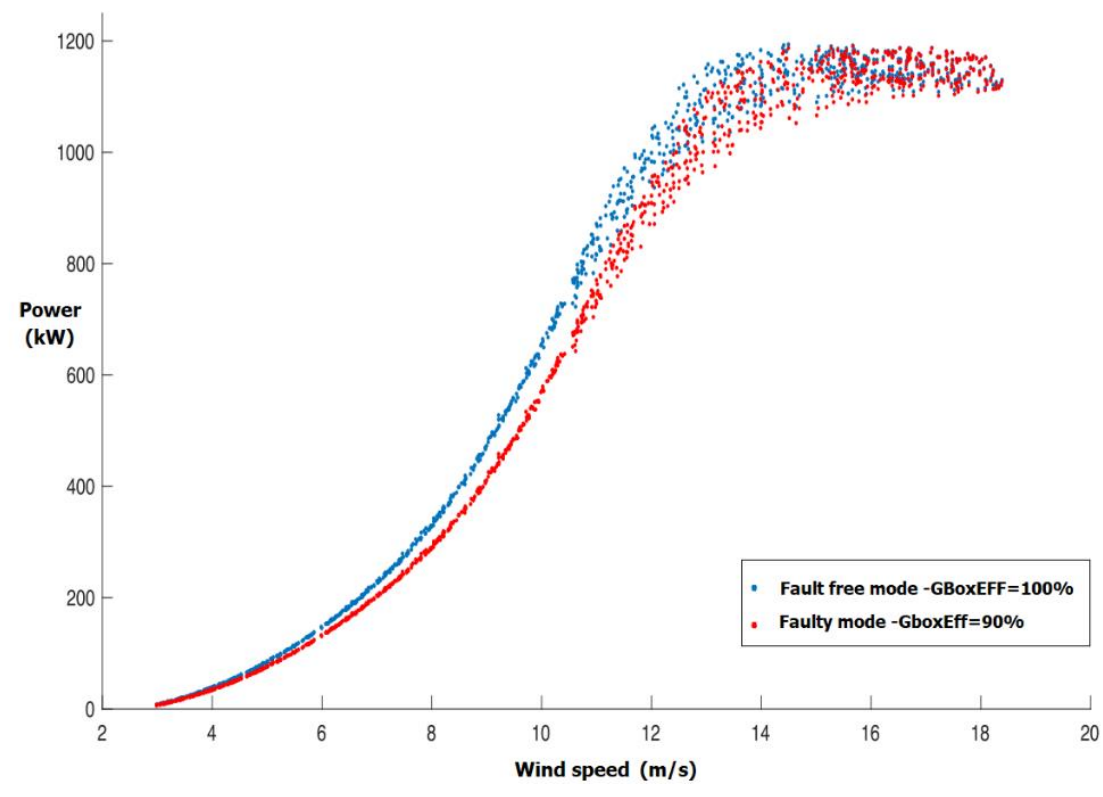

Figure 4

Sample of power curve's scatter plot from raw SCADA data for both fault-free and faulty operating modes

\subsection{Mathematical Modeling and Parameters Estimation}

To study the system's behavior, we need to generate the system's residuals. The least-squares (LS) algorithm is used to estimate the model that describes the system (wind turbine power curve) and to generate the system's residual in addition to study the behavior of the system according to the obtained artificial SCADA data from FAST-AeroDyn Simulator (experimental data). Likewise, according to the experimental data, a model that describes the system behavior was formulated to explain the experimental data and allowed to make predictions of the future responses of the system (WT power curve). Consequently, systematic procedures were applied to generate the system's residuals preparing for the fault detection test.

\section{WT's Power Curve Model Formulation}

A parametric model of WT's power curve is chosen to describe the system. Parametric models assume some finite set of parameters $(\theta)$, given the parameters, future predictions $(\hat{p})$ which are independent of the experimental data 
$(p)$. These parameters are usually collected from a single parameter vector $\theta=\left[\theta_{1} \theta_{2} \ldots \theta_{n}\right][35]$.

\section{WT Power Curve Model Structure}

Polynomial expressions of different orders (degrees) are widely used in many works found in the literature [36] to model the WT's power curve. In this research, a polynomial model was used to describe the WT's power curve as a simple empirical model.

Given the power curve of $\mathrm{m}$ data pairs of power $\left(p 1, p 2, \ldots . ., p_{m}\right)$ versus wind speed $\left(w_{1}, w_{2}, \ldots ., w_{m}\right)$, an $\mathrm{n}^{\text {th }}$ order polynomial was defined as follows:

$$
\hat{p}(w)=a_{1} w^{n}+a_{2} w^{n-1}+\cdots+a_{n} w+a_{n+1}
$$

It is worth mentioning that the fitted model is linear in its parameters.

The main objective is to minimize the least square error between the fitted value and the actual value as:

$$
\left\{a_{l}\right\}=\arg \min \sum_{j=1}^{m}\left\{\left(p_{j}-\hat{p}\left(w_{j}\right)\right)^{2}\right\}
$$

where $\left\{a_{l}\right\}$ is the set of polynomial coefficients, $l \in[1 ; \mathrm{n}+1]$.

\section{Modeling assumptions and parameter estimation}

To fulfill the abovementioned objective, the LS algorithm was used to fit the power curve data and generate residual from the fitted polynomial model (residual generator). The LS algorithm estimates the coefficients of the model (parameters) by minimizing the summation of squares of the residuals.

\subsection{Power Residuals Generation}

Residuals can be calculated as the following:

$$
p_{i}-\hat{p}_{i}
$$

Where $r_{i}$ is the power residual, $p_{i}$ is the actual power, and $\hat{p}_{i}$ is the estimated power based on the power curve model.

Figure 5 depicts a sample of power residuals generated from the estimated $6^{\text {th }}$ degree polynomial model for fault-free operating mode $(k \leq 1000)$ and faulty operating mode $(k>1000)$. In faulty mode, the wind speed is increasing from 2 $\mathrm{m} / \mathrm{s}$ to $18 \mathrm{~m} / \mathrm{s}$, and then it is reversed from $18 \mathrm{~m} / \mathrm{s}$ to $2 \mathrm{~m} / \mathrm{s}$ (to avoid the abrupt degradation in wind speed). The right side of the figure (i.e., $k>1000$ ) shows faulty mode with GBoxEff $=90 \%$ where wind speed is changing from $18 \mathrm{~m} / \mathrm{s}$ to 2 $\mathrm{m} / \mathrm{s}$. 
Note that in the case of the fault-free operating mode ( $k \leq 1000$ in Figure 5), the variance of the first 400 points is smaller than the rest. This set of points are located within a low wind speed area in which the residuals have a small magnitude (superimposed). In the case of the faulty operating mode $(k>$ 1000 in Figure 5), it is the opposite: the variance of the last 400 points is smaller.

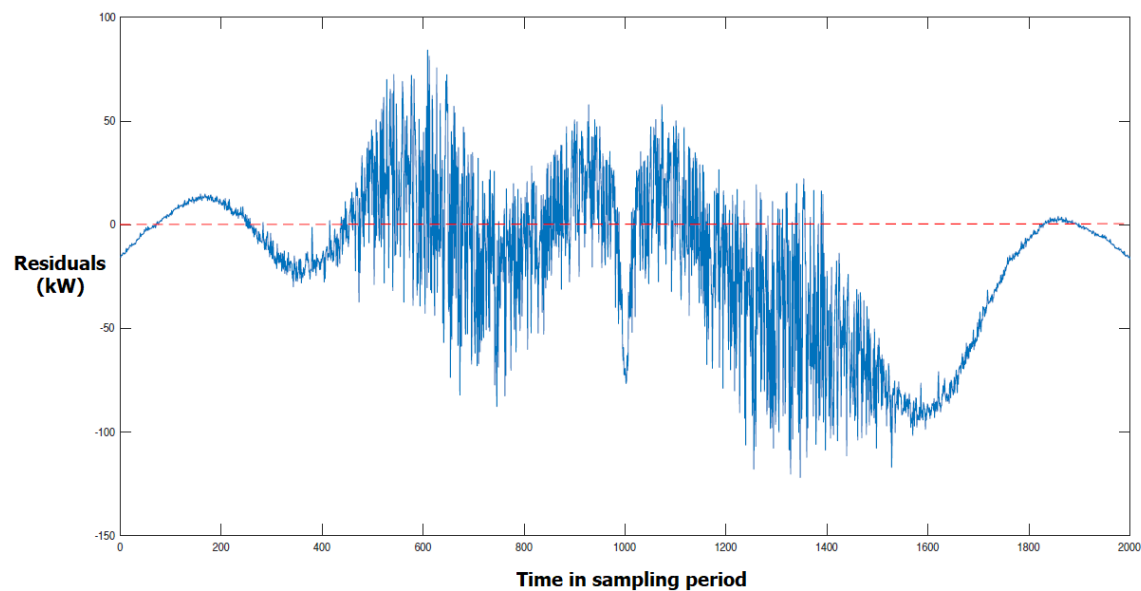

Figure 5

Sample from the generated residuals - fault-free and $90 \%$ GBoxEff faulty mode- time on the $\mathrm{x}$-axis is expressed as the number of samples

\subsection{Model Validation}

The regression model was validated (limited validation within the same data set) by analyzing the goodness of fit of the regression by calculating the mean squared error (MSE) between the actual (experimental) data and the fitted model (measured).

The MSE is a measure of the quality of an estimator, it is always positive, and the smaller value is, the better.

$$
M S E=\frac{1}{n} \sum_{i=1}^{n}\left(p_{i}-\hat{p}_{i}\right)^{2}
$$

Where $n$ is the number of data points of the power, $p_{i}$ is the actual power (experimental) and $\hat{p}_{i}$ is the estimated (measured) power.

The MSE of the fitted polynomial models with order (degree) 3 up to order 6 are illustrated in Table 3, the smaller MSE between the experimental data and the fitting is selected.

Thus, a $6^{\text {th }}$-degree polynomial model is the best fit for the experimental data and could be considered an accepted model. 
Table 3

Best fit -polynomial degree

\begin{tabular}{|c|c|c|c|c|}
\cline { 2 - 5 } \multicolumn{1}{c|}{} & \multicolumn{4}{c|}{ Polynomial degree } \\
\hline $\begin{array}{c}\text { Fault-free operating mode } \\
\text { GBoxEff 100\% }\end{array}$ & $3^{\text {rd }}$ & $4^{\text {th }}$ & $5^{\text {th }}$ & $6^{\text {th }}$ \\
\hline MSE & 1657.3 & 1339.5 & 765.1 & 651.7 \\
\hline
\end{tabular}

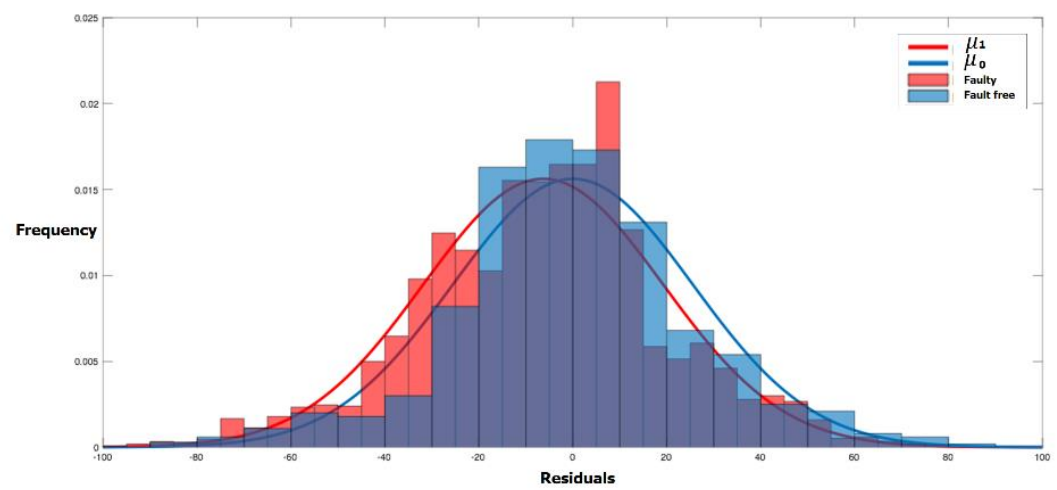

Figure 6

Superposition of the fault-free residuals in blue and the faulty residuals in red

Power residuals are assumed "approximately" to be normally distributed. The histogram in Figure 6 shows the superposition of the fault-free residuals in blue and the faulty residuals in red.

\subsection{Mathematical Model of the Identified System}

Referring to equation 4 , the $6^{\text {th }}$-degree polynomial model that fit the experimental data of the fault-free operational mode of the WT is considered as the first developed model in this research and described below as a function of wind speed:

$\hat{p}\left(w_{i}\right)=0.0012 w_{i}{ }^{6}-0.0523 w_{i}{ }^{5}+0.7154 w_{i}{ }^{4}-3.0313 w_{i}{ }^{3}+$ $5.3390 w_{i}^{2}+3.2694 w_{i}+1.2002$

Where: $\hat{p}\left(w_{i}\right)$ is the measured power, and $w_{i}$ is the wind speed.

Figure 7 shows the estimated $6^{\text {th }}$ degree polynomial model. 


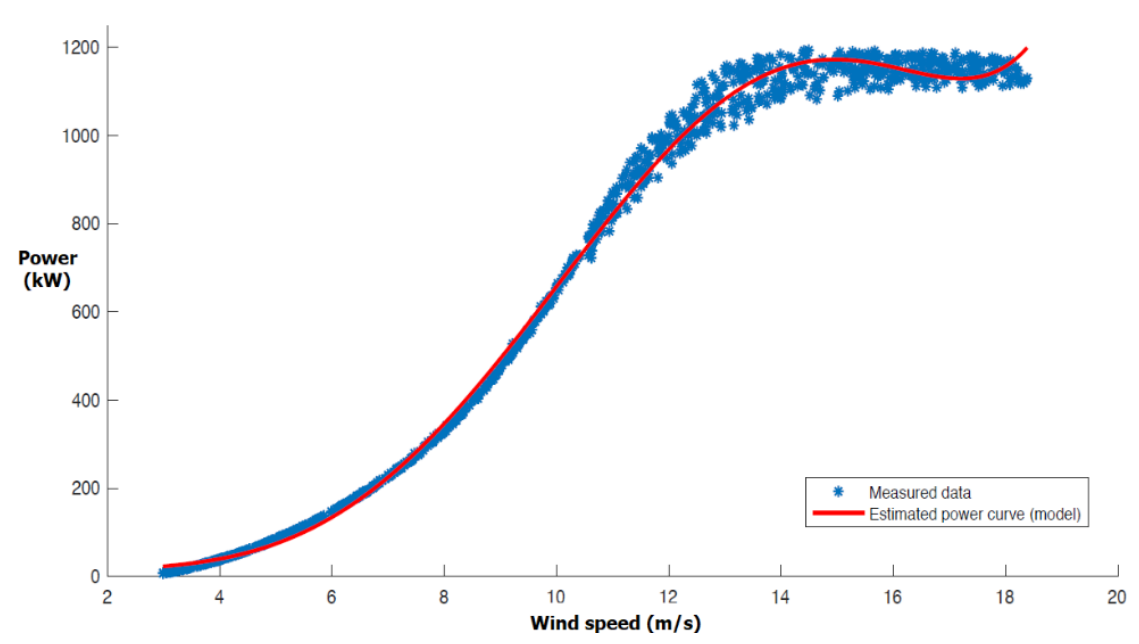

Figure 7

Power curve estimated model: fault-free mode $(\mathrm{GBoxEff}=100 \%)$

\subsection{Tuned Model toward the Nominal Power Curve}

Referring to the manufacturer's nominal power curve (Figure 3), the rated power at the nominal wind speed $(12.5 \mathrm{~m} / \mathrm{s})$ should be a constant.

Thus, a new (tuned) power curve model depicted in Figure 8 is formulated to be more consistent with the manufacturer's nominal power curve at power limitation or constant power region (see region $\mathrm{C}$ in Figure 3).

To achieve this, a non-linear optimization fit was performed through constrained function minimization, as depicted in Figure 8. The polynomial coefficients can be determined as follows:

$$
\left\{a_{l}\right\}=\min \sum_{i=1}^{608}\left(p_{i}-\hat{p}\left(w_{i}\right)\right)^{2}
$$

Subject to:

$$
\hat{p}(12.5)=P_{r}
$$

where $\left\{a_{l}\right\}$ is the set of polynomial coefficients, $l \in[1 ; 7]$, and $P_{r}$ is the rated power.

The optimization problem in (9) was simplified using a penalty function to convert the constrained problem into an unconstrained problem. The general technique is to add a component to the objective function that produces a high cost if the constraint is violated, as is indicated in equation (10). 


$$
\left\{a_{i}\right\}=\min \sum_{i=1}^{608}\left(p_{i}-\hat{p}\left(w_{i}\right)\right)^{2}+\lambda\left|P_{r}-\hat{p}(12.5)\right|
$$

Where $\lambda$ is the penalty coefficient, and point $(i=608)$ is the corresponding point of the fault-free mode in which the wind speed $\left(w_{i}\right)$ is equal to $12.5 \mathrm{~m} / \mathrm{s}$ where the constant power generation (region $\mathrm{C}$ ) starts.

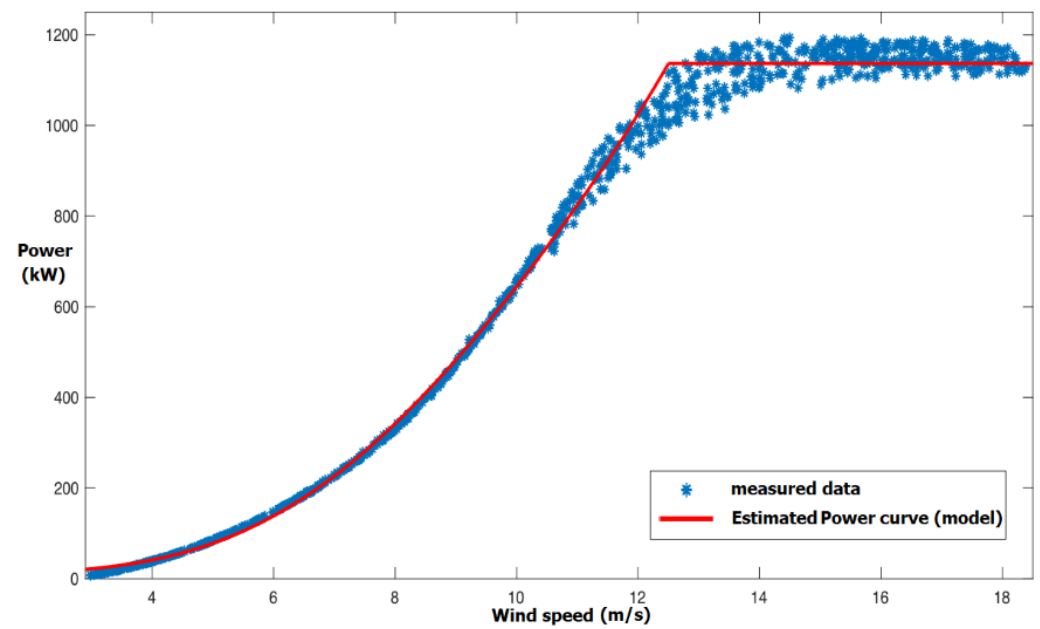

Figure 8

Tuned power curve estimated model based on the nominal power curve

\section{Fault Detection: CUSUM Statistical Change Detection Algorithm}

The generated residuals are evaluated using an on-line CUSUM statistical change detection algorithm to perform the fault detection (performance degradation).

\subsection{Residual Evaluation}

The general purpose of a cumulative summation (CUSUM) test is to test two hypotheses, $\mathcal{H}_{0}$ and $\mathcal{H}_{1}$, against each other to determine which of them describes the data. In our study, $\mathcal{H}_{0}$ and $\mathcal{H}_{1}$ represent the fault-free and faulty operating modes, respectively.

In order to detect small changes in the power residuals, the CUSUM statistical change detection algorithm was used for every single power residual point. 
The power residuals vector was not evaluated at once due to the changes in the off-diagonal values of the residual covariance matrix, which are changing significantly over time; thus, the matrix should be updated continuously. To avoid this, positive change detection in the residuals' mean was applied using the CUSUM algorithm for each power residual component [28].

We have a scalar set of power residuals $\{r(1), \ldots ., r(k)\}$ :

Assuming that the power residuals approximately follow a Gaussian (normal) distribution, and the probability distribution function is:

$$
p_{\mu}(z)=\frac{1}{\sigma \sqrt{2 \pi}} \exp ^{-\frac{(r-\mu)^{2}}{2 \sigma^{2}}}
$$

The hypotheses are as the following:

$$
\begin{aligned}
& \mathcal{H}_{0}: r(i) \sim \mathcal{N}\left(\mu_{0}, \sigma^{2}\right) \text { for } i=(1, \ldots, k) \\
& \mathcal{H}_{1}: r(i) \sim \mathcal{N}\left(\mu_{0}, \sigma^{2}\right) \text { for } i=\left(1, \ldots, k_{0}\right), r(i) \sim \mathcal{N}\left(\mu_{1}, \sigma^{2}\right) \text { for } i=\left(k_{0}, \ldots, k\right)
\end{aligned}
$$

Where:

- $k_{0}$ is the unknown change time

- $\quad \mu_{0}$ and $\mu_{1}$ are the residual means before and after the possible change

The corresponding log-likelihood ratio $s(i)$ for detecting a change in the residual mean from $\mu_{0}$ and $\mu_{1}$ can be calculated as the following:

$$
s(i)=\frac{\mu_{1}-\mu_{0}}{\sigma^{2}}\left(r(i)-\frac{\mu_{1}+\mu_{0}}{2}\right)
$$

Where $\beta=\mu_{1}-\mu_{0}$ is the change in the mean and $b=\frac{\mu_{1}-\mu_{0}}{\sigma}$ is the signal-tonoise ratio.

Figure 9 depicts (radical degradation at gearbox efficiency) the corresponding $\log$-likelihood ratio $s(i)$ for the residuals with $\mu_{0}=0.0043$ before $k=1000$, $\mu_{1}=-34.146$ after $k=1000, \sigma=25.5353$ calculated from equation (12).

Note that the typical behavior of the log-likelihood ratio $s(i)$ shows a negative drift before the change (before $k=1000$ ), and a positive drift after the change (after $k=1000$ ) as depicted in Figure 9. 


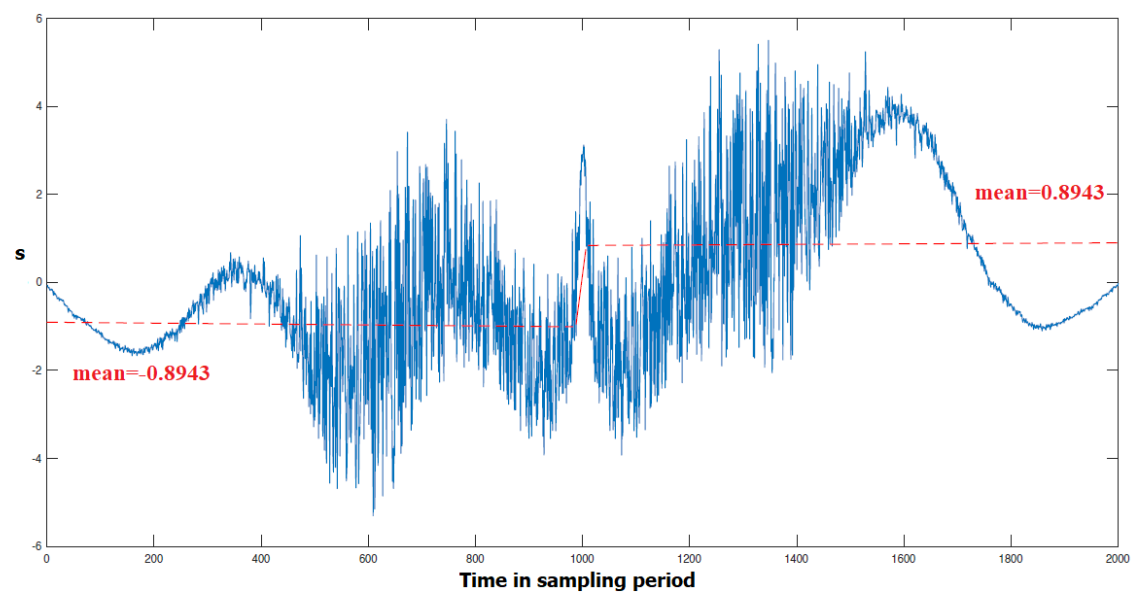

Figure 9

Log-Likelihood ratio of the residuals (residuals realization), radical GBoxEff degradation, time on the $\mathrm{X}$-axis is expressed as the number of samples

The recursive form of the CUSUM algorithm is an efficient and practical way to implement the CUSUM algorithm. Depending on the fact that the threshold ' $h$ ' is always positive, only the contributions to the cumulative sum that add up to a positive number must be considered to determine the decision function [37].

The recursive calculation of the decision function is as the following:

$$
g(k)=\max (0 ; g(k-1)+s(k))
$$

And the alarm function is:

$$
d(k)= \begin{cases}1, & \text { if } g(k)>h \\ 0, & \text { otherwise }\end{cases}
$$

The recursive CUSUM test was implemented on the residuals' sets and on two levels; level one represents the progressive (slight) degradation in gearbox efficiency (GBoxEff: 100-99-98-97\% respectively), and the other level represents the radical degradation in gearbox efficiency (GBoxEff: 100-90\% at once).

A user-defined threshold was defined to avoid or reduce the false alarm and missed detection due to the variations of the parameters. The user-defined threshold needs to consider the maximum magnitudes of residuals under the fault-free test. It is defined as follows:

$$
h=1.5 *(\max g(k) \text { before } k=1000)
$$

Where $\mu_{1}$ is the average of the means for faulty modes (99.98 and 97\%).

The stopping time (also called alarm time) $k_{a}$, is the time instant at which $g(k)$ crosses the user-defined positive threshold $h$ :

$$
k_{a}=\min \{k: g(k) \geq h\}
$$


The fault occurrence time $k_{0}$, can be estimated as the time instant $\hat{k}_{0}$ at which $S(k)$ has changed from a negative to a positive slope. It is formally expressed as:

$$
\hat{k}_{0}=k_{a}-N\left(k_{a}\right)
$$

Where $\mathrm{N}(\mathrm{k})$ is the number of successive observations for which the decision function remains strictly positive.

$$
N(k)=N(k-1) 1_{\{\mathrm{g}(\mathrm{k}-1)>0\}}+1
$$

where $1\{x\}$ is the indicator of event $\mathrm{x}$, namely, $1\{x\}=1$ when $\mathrm{x}$ is true, and $1\{x\}$ $=0$ otherwise.

In equation (14), if $g(k)>h$, an alarm is issued, and an estimate of the change occurrence time $\hat{k}_{0}$ is provided by equation (17), and the decision function is reinitialized to 0 .

The re-initialization after an alarm allowed us to check whether the change in the mean persists as time elapses or not. The result is a sequence of alarm time instants $k_{a}$ and estimated change occurrence times $\hat{k}_{0}$ for increasing time horizon $k$.

Figures 10 and 11 below depict the evolution of the recursive CUSUM decision function with re-initialization when an alarm has been issued for the radical and progressive degradation with $\mu_{0}$ before $k=1000$, and with $\mu_{1}$ after $k=1000$.

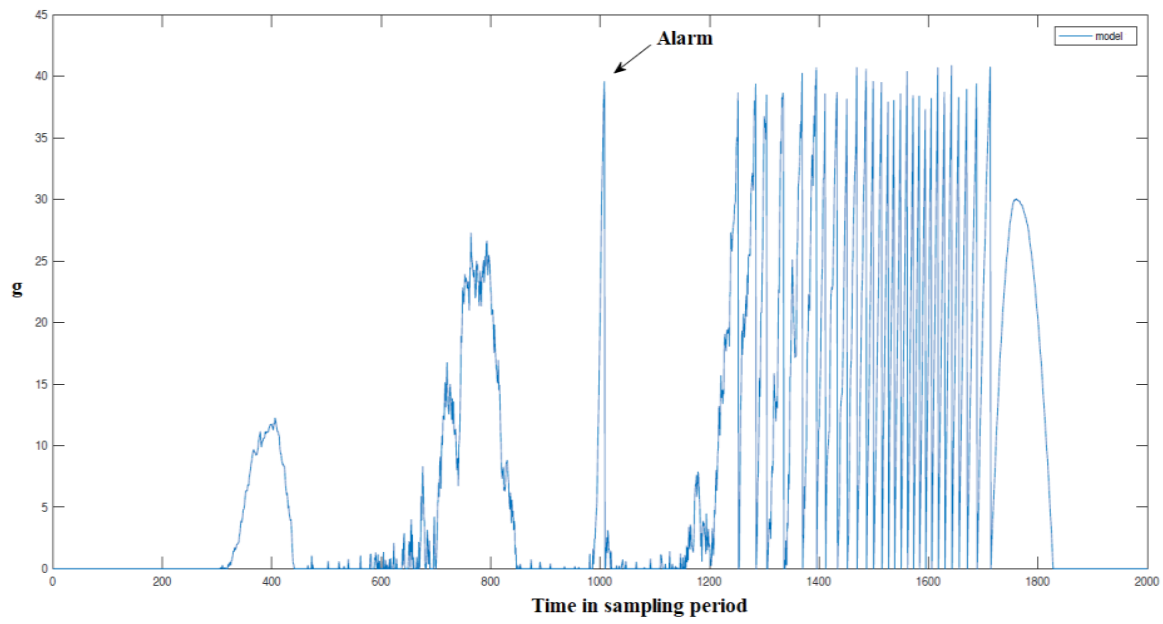

Figure 10

Evolution of the recursive CUSUM decision functions with re-initialization (radical degradation, GBoxEff $=100-90 \%$ ), time on the $\mathrm{x}$-axis is expressed as the number of samples 
For the case of radical gearbox efficiency degradation; from equation (16), the stop alarm is $k_{a}=1009$, while from equations (17) and (18), the fault occurrence time estimate is $\hat{k}_{0}=986$.

In Figure 10, it can be seen that there is a regular cross of the threshold started from $k=1235$, due to the relatively large signal-to-noise ratio; this indicates that a permanent failure has occurred.

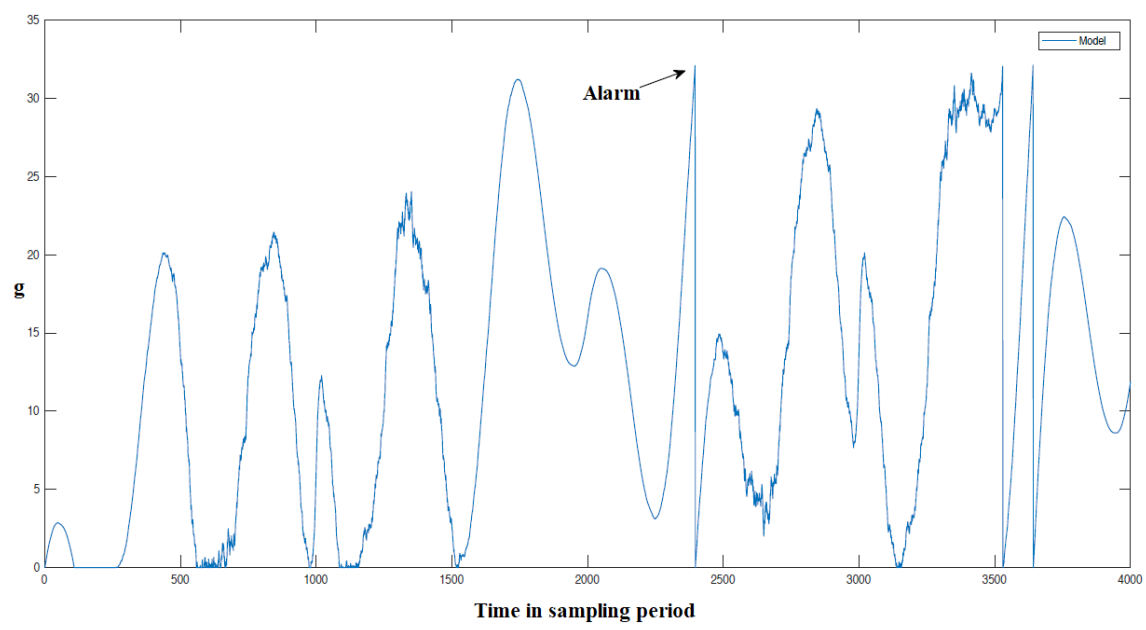

Figure 11

Evolution of the recursive CUSUM decision functions with re-initialization (progressive degradation, GBoxEff $=100-99-98-97 \%$ ), time on the $\mathrm{x}$-axis is expressed as the number of samples

The threshold value $h$ is calculated for each case according to equation (15).

Similarly, for the level of progressive gearbox efficiency degradation, the stop alarm is $k_{a}=2397$, while the fault occurrence time estimate is $\hat{k}_{0}=1524$. There is no regular cross of the threshold because the signal-to-noise ratio is small; accordingly, the algorithm needs more time for the detection.

As shown in Figures 10 and 11, the CUSUM of the log-likelihood ratio is increasing during the fault occurrence. A more increment (higher slope) indicates a larger fault magnitude presence (larger percent of degradation in GBoxEff). At the time when the GBoxEff changed from fault-free mode to faulty mode, i.e., $k=1000$, there is a detection time delay; this delay may occur due to the value of threshold $h$.

Detection time delay is expressed as the number of samples; for the radical GBoxEff degradation, it is 9, while for progressive GBoxEff degradation, it is 1397. This could be interpreted by a high value of threshold $h$. At the same time, if we set the threshold to a lower value, there may be issues of false alarms. It indicates that there is a trade-off between false alarms and detection time. 
From Figures 10 and 11 it can be concluded that the larger the fault (larger percent of degradation in GBoxEff), the shorter the detection time.

\section{Conclusions and Future Work}

In this research, an early fault detection model for the Wind Turbine gearbox, was presented. The power residuals of the model were assumed to be normally distributed; the small deviations residuals should not affect the detection. As found in the literature, the CUSUM algorithm has high robustness to nonnormality, and it is effective in detecting the changes in all sizes, even in highly skewed and heavy-tailed process distributions. The results of power residuals' evaluation using the CUSUM algorithm have been observed for the estimated model and all degradation levels. For progressive GBoxEff degradation level, the developed fault detection system was able to detect a fault magnitude of $2 \%$ under realistic wind turbulence. For the radical degradation level, the fault detection time was shorter than the progressive degradation, the larger the fault, the shorter the detection time.

Thus, it can be concluded that the artificial SCADA data simulated by FAST_NREL, could be used as an efficient source of measurements with the absence of well-documented real SCADA data to study the behavior of a specific component of WT and to perform model-based fault detection, then to help to understand the fault effects. This allows better planning for maintenance activities and gives a better opportunity to implement a predictive maintenance strategy, that can result in the reduction of maintenance costs and increase the reliability of the WT.

This work was validated for one fault type, and one fault indicator was analyzed in the gearbox based on the power curve. This can be used as a framework in the future, to develop a model-based fault detection in WT's gearbox and to include other fault indicators, which could affect the WT's performance and predict, for instance, the remaining useful life (RUL) of the gearbox component, as a fault indicator. Further work can be done by considering a full fault diagnosis study, that includes, fault detection, isolation and estimation. In addition, the proposed model can be validated and the efficiency can be tested on real SCADA data. Moreover, as future work, power curve parameters and fault detection could be predicted using machine learning algorithms, such as ANN, for more prediction accuracy when dealing with a large amount of data or more complex systems. As a result, full implementation of a predictive maintenance strategy, could be carried out, based on this research, aimed at the development of even better maintenance planning and any associated scheduling activities.

\section{Acknowledgment}

The authors would like to thank the Department of Control Engineering and System Analysis at the Free University of Brussels (ULB) and the head of department Prof. Michel Kinnaert for funding and supervising this research. 


\section{References}

[1] Global Wind Energy Council, "Global Wind Report 2019," 2019

[2] IEA, "World Energy Outlook 2019 - Analysis," 2019

[3] P. Kádár, "Pros and cons of the renewable energy application," Acta Polytechnica Hungarica, Vol. 11, No. 4, pp. 211-224, 2014, DOI: 10.12700/aph.25.04.2014.04.14

[4] M. Monfared, H. Rastegar, and H. M. Kojabadi, "A new strategy for wind speed forecasting using artificial intelligent methods," Renewable Energy, Vol. 34, No. 3, pp. 845-848, Mar. 2009, DOI: 10.1016/j.renene.2008.04.017

[5] B. Abichou, D. Flórez, M. Sayed-mouchaweh, H. Toubakh, B. François, and N. Girard, "Fault Diagnosis Methods for Wind Turbines Health Monitoring: a Review," European Conference of the Prognostics and Health Management Society 2014, no. August, pp. 1-8, 2013, DOI: $10.13140 / 2.1 .1150 .6563$

[6] J. Feng and W. Z. Shen, "Design optimization of offshore wind farms with multiple types of wind turbines," Applied Energy, Vol. 205, no. August, pp. 1283-1297, 2017, DOI: 10.1016/j.apenergy.2017.08.107

[7] M. Carlberg, "Quantify Change in Wind Turbine Power Performance Using Only SCADA Data," KTH, 2015

[8] P. Tchakoua, R. Wamkeue, M. Ouhrouche, F. Slaoui-Hasnaoui, T. Tameghe, and G. Ekemb, "Wind Turbine Condition Monitoring: State-ofthe-Art Review, New Trends, and Future Challenges," Energies, Vol. 7, No. 4, pp. 2595-2630, Apr. 2014, DOI: 10.3390/en7042595

[9] T. Yuan, Z. Sun, and S. Ma, "Gearbox fault prediction of wind turbines based on a stacking model and change-point detection," Energies, Vol. 12, No. 22, 2019, DOI: 10.3390/en12224224

[10] B. Hahn, M. Durstewitz, and K. Rohrig, "Reliability of Wind Turbines: Experiences of 15 years with 1,500 WTs," Wind Energy: Proceedings of the Euromech Colloquium, no. July, pp. 329-332, 2007, DOI: 10.1007/978-3-540-33866-6

[11] P. Santos, L. F. Villa, A. Reñones, A. Bustillo, and J. Maudes, "An SVMbased solution for fault detection in wind turbines," Sensors (Switzerland), Vol. 15, No. 3, pp. 5627-5648, 2015, DOI: 10.3390/s150305627

[12] L. Wenyi, W. Zhenfeng, H. Jiguang, and W. Guangfeng, "Wind turbine fault diagnosis method based on diagonal spectrum and clustering binary tree SVM," Renewable Energy, Vol. 50, pp. 1-6, 2013, DOI: 10.1016/j.renene.2012.06.013 
[13] P. Bangalore and L. B. Tjernberg, "An approach for self evolving neural network based algorithm for fault prognosis in wind turbine," 2013, DOI: 10.1109/PTC.2013.6652218

[14] H. Wang, H. Wang, G. Jiang, J. Li, and Y. Wang, "Early fault detection of wind turbines based on operational condition clustering and optimized deep belief network modeling," Energies, Vol. 12, No. 6, 2019, DOI: 10.3390/en12060984

[15] P. Bangalore, S. Letzgus, D. Karlsson, and M. Patriksson, "An artificial neural network-based condition monitoring method for wind turbines, with application to the monitoring of the gearbox," Wind Energy, Vol. 20, No. 8, pp. 1421-1438, Aug. 2017, DOI: 10.1002/we.2102

[16] K. T. Abd-Elwahab and A. A. Hassan, "SCADA data as a powerful tool for early fault detection in wind turbine gearboxes," Wind Engineering, Vol. 1, No. 10, Dec. 2020, DOI: 10.1177/0309524X20969418

[17] S. Butler, F. O'connor, D. Farren, and J. V Ringwood, "A feasibility study into prognostics for the main bearing of a wind turbine," 2012, DOI: 10.0/Linux-x86_64

[18] B. Chen, D. Zappalá, C. J. Crabtree, and P. J. Tavner, "Survey of Commercially Available SCADA Data Analysis Tools for Wind Turbine Health Monitoring," 2014

[19] L. Tao, Q. Siqi, Y. Zhang, and H. Shi, "Abnormal Detection of Wind Turbine Based on SCADA Data Mining," Mathematical Problems in Engineering, Vol. 2019, 2019, DOI: 10.1155/2019/5976843

[20] B. Corley, S. Koukoura, J. Carroll, and A. McDonald, "Combination of Thermal Modelling and Machine Learning Approaches for Fault Detection in Wind Turbine Gearboxes," Energies, Vol. 14, No. 5, p. 1375, 2021, DOI: $10.3390 /$ en 14051375

[21] M. Schlechtingen, I. F. Santos, and S. Achiche, "Wind turbine condition monitoring based on SCADA data using normal behavior models. Part 1: System description," Applied Soft Computing Journal, Vol. 13, No. 1, pp. 259-270, Jan. 2013, DOI: 10.1016/j.asoc.2012.08.033

[22] M. Schlechtingen and I. F. Santos, "Wind turbine condition monitoring based on SCADA data using normal behavior models. Part 2: Application examples," Applied Soft Computing Journal, Vol. 14, no. PART C, pp. 447-460, Jan. 2014, DOI: 10.1016/j.asoc.2013.09.016

[23] W. Yang, R. Court, and J. Jiang, "Wind turbine condition monitoring by the approach of SCADA data analysis," Renewable Energy, Vol. 53, pp. 365-376, May 2013, DOI: 10.1016/j.renene.2012.11.030 
[24] A. Zaher, S. D. J. McArthur, D. G. Infield, and Y. Patel, "Online wind turbine fault detection through automated SCADA data analysis," Wind Energy, Vol. 12, No. 6, pp. 574-593, Sep. 2009, DOI: 10.1002/we.319

[25] Z. Y. Zhang and K. S. Wang, "Wind turbine fault detection based on SCADA data analysis using ANN," Advances in Manufacturing, Vol. 2, No. 1, pp. 70-78, Mar. 2014, DOI: 10.1007/s40436-014-0061-6

[26] J. L. Godwin and P. C. Matthews, "Classification and detection of wind turbine pitch faults through SCADA data analysis," International Journal of Prognostics and Health Management; Special Issue Wind Turbine PHM, Vol. 4, 2013

[27] A. Kusiak and W. Li, "The prediction and diagnosis of wind turbine faults," Renewable Energy, Vol. 36, No. 1, pp. 16-23, Jan. 2011, DOI: 10.1016/j.renene.2010.05.014

[28] A. B. Borchersen and M. Kinnaert, "Model-based fault detection for generator cooling system in wind turbines using SCADA data," Wind Energy, Vol. 19, No. 4, pp. 593-606, Apr. 2016, DOI: 10.1002/we.1852

[29] G. Pujol-Vazquez, L. Acho, and J. Gibergans-Báguena, "Fault detection algorithm for wind turbines' pitch actuator systems," Energies, Vol. 13, No. 11, 2020, DOI: 10.3390/en13112861

[30] B. J. Jonkman and M. L. Buhl, "TurbSim User's Guide," 2006

[31] J. M. Jonkman, G. J. Hayman, B. J. Jonkman, and R. R. Damiani, "AeroDyn v15 User's Guide and Theory Manual," 2006

[32] J. M. Jonkman and M. L. Buhl, "FAST User's Guide," 2005

[33] U. Eminoglu and S. Ayasun, "Modeling and design optimization of variable-speed wind turbine systems," Energies, Vol. 7, No. 1, pp. 402419, 2014, DOI: 10.3390/en7010402

[34] I. E. commission (IEC), "IEC 61400-1:2019, international standard - wind turbine," 2019

[35] O. Uluyol, G. Parthasarathy, W. Foslien, and K. Kim, "Power curve analytic for wind turbine performance monitoring and prognostics," in Proceedings of the Annual Conference of the Prognostics and Health Management Society 2011, PHM 2011, 2014, pp. 435-442

[36] M. Lydia, S. S. Kumar, A. I. Selvakumar, and G. E. Prem Kumar, "A comprehensive review on wind turbine power curve modeling techniques," Renewable and Sustainable Energy Reviews, Vol. 30, pp. 452-460, Feb. 2014, DOI: 10.1016/j.rser.2013.10.030

[37] M. Blanke, M. Kinnaert, J. Lunze, and M. Staroswiecki, Diagnosis and Fault-Tolerant Control. Berlin, Heidelberg: Springer Berlin Heidelberg, 2003 